\title{
NOTE ON THE STRUCTURE OF GRAPHS
}

\author{
G. A. Dirac
}

(received October 10, 1961)

1. Introduction. This paper is concerned with undirected graphs which may be infinite and may contain multiple edges. The Axiom of Choice is assumed. The terms path, infinite path and circuit are used in the same sense as Weg, unendlicher Weg and Kreis, respectively, a re used in D. Konnig's book [1]. The valency of a vertex is the number of edges incident with it. The length of a path is the number of edges in it.

The following theorem is a generalization of the well known fact that if a vertex of a graph is not a cut-vertex (Artikulation [2]) and has valency $\geqq 2$, then the graph contains at least one circuit to which the vertex belongs.

THEOREM A. If the vertex a of a graph is not a cut-vertex and has valency $\geqq 2 \epsilon$, where $\epsilon$ is an integer, then the graph contains $\epsilon$ circuits each of which passes through $a$, no two of which have an edge in common, and any two of which have at most one vertex besides a in common.

A simple example is furnished by a graph consisting of the two vertices $\underline{a}$ and $\underline{b}$ joined by $2 \epsilon$ edges.

2. Disentangling pairings. Let $\Gamma$ be a graph and let $V$ denote a set containing an even number of vertices belonging to $\Gamma$. A partition of $\mathrm{V}$ into (mutually disjoint) pairs will be called a disentangling pairing of $\mathrm{V}$ with respect to $\Gamma$ if $\Gamma$ contains a set of paths with the properties that corresponding to each pair of the partition there is a path of the set having the two vertices of the pair as its two end-vertices, and no two paths of the set have more than one vertex in common.

Canad. Math. BuIl. vol. 5, no. 3, September 1962. 
THEOREM B. If $\Gamma$ is a connected graph and $V$ is a set containing an even number of the vertices of $\Gamma$ then there exists a disentangling pairing of $V$ with respect to $\Gamma$.

Proof. Let $|\mathrm{V}|=2 v$. Corresponding to an arbitrary partition $P$ of $V$ into $v$ pairs, $\Gamma$ contains a set of $v$ paths such that corresponding to each pair of $P$ there is a path of the set having the two vertices of the pair as its endvertices; for $\Gamma$ is connected, and among all such sets of paths there exists at least one set having the property that the sum of the lengths of the $v$ paths in it is minimal. Let $L(P)$ denote this minimal sum. Among all possible partitions of $V$ into pairs one may be selected, $P$ say, with the property that $L\left(P_{0}\right)$ is minimal. Let $\left\{v_{1}{ }_{v_{1}}^{O}\right\}, \ldots,\left\{v_{v} v_{v}{ }^{\prime}\right\}$ be the $v$ pairs of $P_{0}$ and $r_{1}, \ldots, r_{v}$ be $v$ paths contained in $\Gamma$ such that $r_{i}$ has $v_{i}$ and $v_{i}{ }^{\prime}$ as its end-vertices for $i=1, \ldots, v$ and the sum of the Iengths of the $v$ paths is $L\left(P_{0}\right)$.

No two of $r_{1}, \ldots, r_{\nu}$ have more than one vertex in common. Proof: suppose on the contrary that e.g., $r_{1}$ and $r_{2}$ have more than one vertex in common. Going along $r_{2}$ from $v_{2}$ to $v_{2}^{\prime}$ ' let $x$ be the first and $\underline{y}$ the last vertex of $r_{1}$ encountered, $\underline{x} \neq \underline{y}$ because $r_{1}$ and $r_{2}$ have more than one vertex in common. It may be assumed without loss of generality that going along $r_{1}$ from $v_{1}$ to $v_{1}^{\prime}, \underline{x}$ is encountered before $\underline{y}$. The convention will be adopted that if $r$ is a path and $\underline{m}$ and $\underline{n}$ are vertices of $r$ then $r[m, n]$ denotes that path contained in $r$ which has $\underline{m}$ and $\underline{n}$ as its two end-vertices if $\underline{m} \neq \underline{n}$, and $r[m, n]$ denotes $\underline{m}$ if $\underline{m}=\underline{n}$; $\|r\|$ denotes the length of $r$. In this notation let $r_{v+1}=r_{1}\left[v_{1}, x\right] \cup r_{2}\left[v_{2}, x\right]$ and $r_{v+2}=r_{2}\left[v_{2}{ }^{\prime}, y\right] \cup r_{1}\left[v_{1}{ }^{\prime}, y\right]$. $r_{\nu+1}$ and $r_{\nu+2}$ are paths contained in $\Gamma$ having $v_{1}, v_{2}$ and $\mathrm{v}_{1}{ }^{\prime}, \mathrm{v}_{2}{ }^{\prime}$ respectively as their end-vertices, and $\left\|r_{v+1}\right\|+\left\|r_{v+2}\right\|=\left\|r_{1}\right\|+\left\|r_{2}\right\|-\left\|r_{1}[x, y]\right\|-\left\|r_{2}[x, y]\right\|<\left\|r_{1}\right\|+\left\|r_{2}\right\|$. So if $P_{1}$ denotes the partition obtained from $P_{0}$ by replacing 
the two pairs $\left\{\mathrm{v}_{1}, \mathrm{v}_{1}^{\prime}\right\}$ and $\left\{\mathrm{v}_{2}, \mathrm{v}_{2}{ }^{\prime}\right\}$ by the two pairs $\left\{\mathrm{v}_{1}, \mathrm{v}_{2}\right\}$ and $\left\{\mathrm{v}_{1}{ }^{\prime}, \mathrm{v}_{2}{ }^{\prime}\right\}$ then $\mathrm{L}\left(\mathrm{P}_{1}\right)<\mathrm{L}\left(\mathrm{P}_{\mathrm{O}}\right)$, which contradicts the minimality of $L\left(P_{0}\right)$. This contradiction proves that no two of $r_{1}, \ldots, r_{v}$ have more than one vertex in common.

$P_{0}$ is therefore a disentangling pairing of $V$ with respect to $\Gamma$. This proves Theorem $B$.

3. Proof of Theorem A by induction over $\epsilon$. The theorem is obviously true for $\epsilon=1$ because a is not a cutvertex. Assume that the theorem is true for $\overline{1} \leqq \epsilon<\delta$, and suppose that the vertex a is not a cut-vertex of the graph $\triangle$. Let $E$ be a set of $2 \delta$ edges of $\Delta$ each of which is incident with $\underline{\text { a. }}$

If there are two edges in $E, e_{1}$ and $e_{2}$ say, connecting a with the same vertex of $\Delta, \underline{b}$ say, then by the induction hypothesis $\Delta-\mathrm{e}_{1}-\mathrm{e}_{2}$ (the graph obtained from $\Delta$ by deleting $e_{1}$ and $e_{2}$ ) contains a set of $\delta-1$ circuits with the properties stated in Theorem $A$. These circuits and the circuit $a \cup j \cup e_{1} \cup e_{2}$ together constitute a set of $\delta$ circuits with the required properties.

Suppose that no two edges of $E$ connect a with the same vertex of $\Delta$. Then $\underline{a}$ is joined by edges to a set of $2 \delta$ vertices; Iet $\mathrm{V}$ denote this set. $\Delta$ - a (the graph obtained from $\Delta$ by deleting $a$ and all edges incident with $\underline{\text { a) }}$ is connected since $\underline{a}$ is not a cut-vertex of $\Lambda$. By Theorem $B$ there exists a disentangling pairing of $V$ with respect to $\Delta-a$. Let $r_{1}, \ldots, r_{\delta}$ denote the corresponding paths. For $i=1,2, \ldots, \delta$ let $C_{i}$ denote the circuit obtained by joining a to the two end-vertices of $r_{i}$; clearly $C_{i} \subset \Delta$. Any two of $\mathrm{C}_{1}, \ldots, \mathrm{C}_{\delta}$ have at most one vertex besides a in common and no two of $\mathrm{C}_{1}, \ldots, \mathrm{C}_{\delta}$ have an edge in common; for any two of $r_{1}, \ldots, r_{\delta}$ have at most one vertex in common and no 
two of $r_{1}, \ldots, r_{\delta}$ end in the same vertex. $C_{1}, \ldots, C_{\delta}$ therefore satisfy the conditions of Theorem A. Theorem A is thus true for $\epsilon=\delta$ if it is true for $1 \leq \epsilon<\delta$, and it is true for $\epsilon=1$. Therefore the theorem is true for all finite $\epsilon$. Note: Theorem A clearly remains true if loops a re counted as circuits.

4. Vertices with infinite valency. A theorem will be established from which the analogue of Theorem $A$ for infinite $\epsilon$ follows obviously. It is convenient to distinguish two special type of graphs. An $\underline{x}_{0}$-fence is the union of an infinite path $r$ and an enumerable set of mutually disjoint paths $r_{1}, r_{2}, r_{3}, \ldots$ such that for each $i \geq 1, r \cap r_{i}$ consists of one of the endvertices of $r_{i}$; the other end-vertex of $r_{i}$ is called an end-

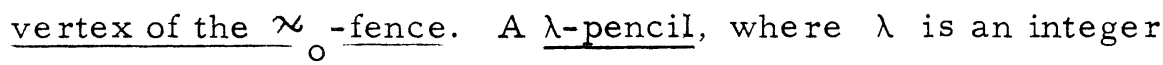
$\geq 3$ or an infinite power, is the union of $\lambda$ distinct paths such that one vertex, called the focus, is end-vertex of all the paths and any two of the paths have the focus and nothing else in common; the other end-vertices of the paths are called endvertices of the $\lambda$-pencil.

THEOREM C. If the vertex a of the graph $\triangle$ is not a cut-vertex of $\lfloor$ and has valency $\geq \epsilon$, where $\epsilon$ is an infinite power, then if $\epsilon>x_{0}$ either $\underline{a}$ is joined to some vertex by at least $\epsilon$ edges or $a$ is joined to every end-vertex of an $\epsilon$-pencil contained in $\Delta-a$; and if $\epsilon=\lambda_{0}$ then either at least one of the alte rnatives just mentioned holds, or $\underline{a}$ is joined to infinitely many vertices of an infinite path contained in $\Lambda$-a, or else $\underline{a}$ is joined to every end-vertex of an $\lambda$-fence containe in $\Delta-\mathrm{a}$.

The analogue of Theorem $\mathrm{A}$ for infinite $\epsilon$ clearly follows from Theorem $\mathrm{C}$.

Theorem C follows from

THEOREM D. Let $\Gamma$ be an infinite connected graph and $\mathrm{V}$ an infinite subset of the set of vertices of $\Gamma$. If $\mathrm{V}$ is enumerable $\Gamma$ either contains a $|V|$-pencil whose end-vertices all belong to $\mathrm{V}$ or an infinite path containing infinitely many 
vertices belonging to $\mathrm{V}$ or an $\varkappa_{0}$-fence whose end-vertices all belong to $\mathrm{V}$; if $\mathrm{V}$ is not enumerable then $\Gamma$ contains a $|\mathrm{V}|$-pencil whose end-vertices all belong to $\mathrm{V}$.

Proof of Theorem D. By transfinite induction or Zorn's Lemma it can be shown that $\Gamma$ contains a connected suingraph $\Gamma$ to which all the vertices of $\mathrm{V}$ belong and which is such that any proper subgraph of $\Gamma^{\prime}$ either does not contain all the vertices, of $\mathrm{V}$ or is disconnected; $\Gamma^{\prime}=\Gamma$ possibly.

$\Gamma$ does not contain a circuit.

For if $\Gamma^{\prime}$ contained a circuit then deleting any one edge of the circuit would leave a connected graph containing all the vertices of $\mathrm{V}$.

Every edge of $\Gamma^{\prime}$ belongs to some path whose endvertices belong to $\mathrm{V}$.

Because if an edge did not have this property then the graph obtained by deleting it from $\Gamma^{\prime}$ would consist of two connected components by (1), and one of the two connected components would contain no vertex belonging to $\mathrm{V}$; this would contradict the minimal property of $\Gamma^{\prime}$.

Corresponding to any two distinct vertices of $\Gamma^{\prime}$ there is a unique path contained in $\Gamma_{1}$ which has them as its endvertices.

For $\Gamma^{\prime}$ is connected and contains no circuit. The length of the path will be called the distance between the two vertices in $\Gamma^{\prime}$.

If $\mathrm{b}$ is any vertex of $\Gamma^{\prime}$ incident with at least three edges of $\Gamma^{\prime}$ then $\Gamma^{\prime}$ contains a pencil which has $b$ as focus, which contains every edge of $\Gamma^{\prime}$ incident with $b$ and whose end-vertices all belong to $\mathrm{V}$.

This follows from (1) and (2).

Suppose that $\mathrm{V}$ is enumerable. If some vertex of $\Gamma$, is incident with infinitely many edges of $\Gamma^{\prime}$, then $\Gamma^{\prime}$ contains 
a $|\mathrm{V}|$-pencil whose end-vertices all belong to $\mathrm{V}$, by (4). If no vertex of $\Gamma$ is incident with infinitely many edges of $\Gamma$ then by a theorem of D. König [3] each vertex of $\Gamma$ is the endvertex of some infirite path contained in $\Gamma$ '. Let $r$ denote an infinite path contained in $\Gamma_{i}$ with the vertex $c$ as endvertex. There are two alternatives: either $r$ contains infinitely many vertices belonging to $\mathrm{V}$ or it does not. If $r$ does not contain an infinite number of vertices belonging to $V$ then, by the minimal property of $\Gamma, \quad r$ contains an infinite number of vertices having valency $\geq 3$ in $\Gamma$; let the se be denoted by $c_{1}, c_{2}, c_{3}, \ldots$ in order along $r$ starting from $c$, and for $i=1,2,3, \ldots$ let $e_{i}$ be an edge incident with $c_{i}$ and not belonging to $r$. By (2) for each $i \geq 1$ there is at least one path contained in $\Gamma$, which has $c_{i}$ as one end-vertex, contains $e_{i}$, and has a vertex belonging to $V$ as its other end-vertex; let such a path be denoted by $r_{i}$. By (1) $r \cup r_{1} \cup r_{2} \cup r_{3} \cup \ldots$ is an $\chi_{0}$-fence whose end-vertices all belong to $V$. This proves Theorem $\mathrm{D}$ for enumerable $\mathrm{V}$.

If $\mathrm{V}$ is not enumerable then $\Gamma$, contains a vertex incident with $|V|$ edges of $\Gamma$. (By (4) no vertex of $\Gamma^{\prime}$ is incident with more than $|V|$ edges of $\Gamma$.) For suppose that every vertex of $\Gamma$, has valency $<|V|$. Let $a_{0}$ denote an arbitrarily chosen vertex of $\Gamma$, and for $i=1,2,3, \ldots$ let $A_{i}$ denote the set of those vertices of $\Gamma^{\prime}$ which are at a distance $i$ from $a_{0}$ in $\Gamma^{1}$. Since the product of two powers each smaller than $|\mathrm{V}|$ is smaller than $|V|$ we have that $\left|A_{i}\right|<|V|$ for $i=1,2,3, \ldots$ and $\left|A_{1} \cup A_{2} \cup A_{3} \cup \ldots\right|<\lambda_{0}|V|$ $|V|$, which is a contradiction. Hence $\Gamma$, contains a vertex incident with $|\mathrm{V}|$ edges of $\Gamma$.

It follows from (4) that $\Gamma$, contains a $|V|$-pencil whose end-vertices all belong to $\mathrm{V}$. This completes the proof of Theorem D.

Proof of Theorem C. Let $V$ denote the set of those vertices of $\Delta$ which are joined to a. Either there exists a vertex joined to $\underline{a}$ by at least $\epsilon$ edges, or else $|\mathrm{V}| \geq \epsilon$. 
If $|\mathrm{V}| \geq \epsilon$ then Theorem $\mathrm{C}$ follows from Theorem $\mathrm{D}$ applied to $\Delta-\mathrm{a}$.

Note. If $a$ is a cut-vertex of $\Delta$ but $\Delta$-a has fewer than $\epsilon$ connected components then the assertion of Theorem $C$ is still true, because at least one of the connected components of $\Delta^{-a}$ has the property that at least $\epsilon$ edges connect a with it.

\section{REFERENCES}

1. D. König, Theorie der endlichen und unendlichen Graphen, Leipzig, 1936.

2. p. 224 .

3. p. 80 , Satz 3 .

Hamilton College

McMaster University 